\title{
ERRATUM
}

J.-S. Zhao - K. Zhou - Z.-J. Feng - Z.-Y. Tan

\section{The singularity study of spatial hybrid mechanisms based on screw theory}

Published online: 16 June 2004

(C) Springer-Verlag London Limited 2004

In the right column after Fig. 8, two equations and the accompanying sentence were published incorrectly. They should read:

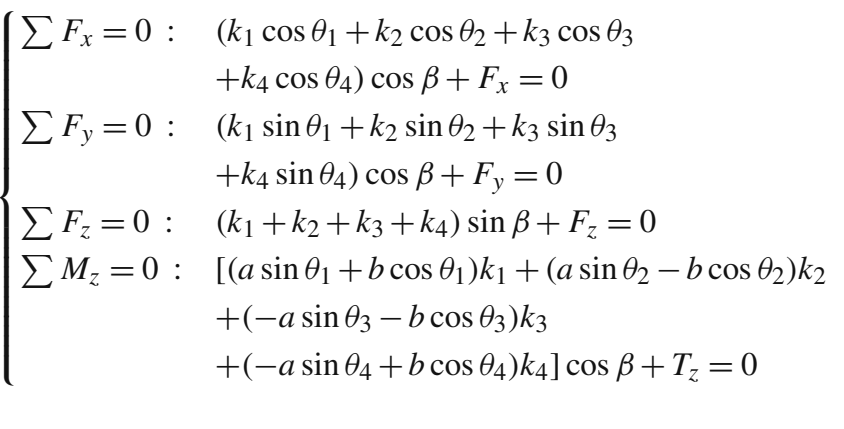

J.-S. Zhao ( · K. Zhou · Z.-J. Feng · Z.-Y. Tan

Department of Precision Instruments, Tsinghua University,

Beijing 100084, P.R. China

E-mail: zjs01@mails.tsinghua.edu.cn

Fax: +86-01-062782351 where $\theta_{i}(i=1,2,3,4)$ denotes the angular coordinate of the projection line of the $i$ th hybrid branch to the $x$-axis in the absolute coordinate system. The above equations can be transformed to:

$A F_{i}=F$

where

$\left.A=\left[\begin{array}{cccc}\cos \theta_{1} & \cos \theta_{2} & \cos \theta_{3} & \cos \theta_{4} \\ \sin \theta_{1} & \sin \theta_{2} & \sin \theta_{3} & \sin \theta_{4} \\ 1 & 1\end{array}\right]\left\{\begin{array}{c}1 \\ \left\{\begin{array}{l}a \sin \theta_{1} \\ +b \cos \theta_{1}\end{array}\right\}\left\{\begin{array}{c}-a \sin \theta_{3} \\ -b \cos \theta_{2}\end{array}\right\}\left\{\begin{array}{c}-a \sin \theta_{4} \\ -b \cos \theta_{3}\end{array}\right\} \\ +b \cos \theta_{4}\end{array}\right\}\right]$.

The online version of the original article can be found at http://dx.doi.org/10.10007/s00170-003-1934-1. 